\title{
Efficacy and safety of different terbinafine regimens in patients of recurrent tinea corporis and cruris
}

\author{
Noopur Verma ${ }^{1 *}$, Savita Verma ${ }^{1}$, Surbhi Dayal' ${ }^{2}$, M. C. Gupta ${ }^{1}$
}

\author{
${ }^{1}$ Department of Pharmacology, ${ }^{2}$ Department of Skin V. D. and Leprosy, Pt. B. D. Sharma PGIMS, Rohtak, Haryana, \\ India
}

Received: 13 June 2021

Revised: 25 June 2021

Accepted: 28 June 2021

*Correspondence:

Dr. Noopur Verma,

Email: verma.noopur3@gmail.com

Copyright: (c) the author(s), publisher and licensee Medip Academy. This is an open-access article distributed under the terms of the Creative Commons Attribution Non-Commercial License, which permits unrestricted non-commercial use, distribution, and reproduction in any medium, provided the original work is properly cited.

\begin{abstract}
Background: Tinea corporis and cruris is said to be recurrent when there is relapse of sign and symptoms after 6 weeks of cure. Recently, there has been increase in cases of recurrent tinea corporis and cruris, with atypical lesions. This study was done to establish efficacy and safety of different terbinafine regimens against recurrent tinea corporis and cruris.

Methods: Sixty patients with clinically and mycologically diagnosed recurrent tinea corporis and cruris were enrolled and divided into three groups. Group A was administered oral terbinafine $500 \mathrm{mg}$ once daily for 2 weeks, group B was given terbinafine $250 \mathrm{mg}$ once daily for double duration i.e., 4 weeks, and group $\mathrm{C}$ was given standard treatment which is $250 \mathrm{mg}$ once daily for 2 weeks. Physician assessment four-point scale (PA4PS) and KOH wet mount were assessed for clinical and mycological efficacy. Biochemical laboratory parameters (liver function tests and kidney function tests) and adverse drug reactions were assessed for safety.

Results: At the end of 6 weeks, reduction in PA4PS from baseline was 46.5\%, 95.8\%, and 20.4\% in groups with double dose, double duration and standard therapy respectively with statistically significant $(\mathrm{p}<0.05)$ improvement in group with double duration. Mycological cure at the end of 4 weeks was $80 \%, 100 \%$ and $50 \%$. There was no safety concern in any of the groups.

Conclusions: Double duration of terbinafine was found to be more efficacious and safer.
\end{abstract}

Keywords: Recurrent tinea corporis, Recurrent tinea cruris, Terbinafine, $\mathrm{KOH}$ mount, PA4PS

\section{INTRODUCTION}

Superficial Tinea corporis is said to be recurrent when there is recurrence of the disease within 6 weeks after the completion of treatment. ${ }^{1}$ The prevalence of superficial mycosis is on the rise with change in spectrum of infection and isolation of some uncommon species. ${ }^{2}$ Patients are presenting with atypical, widespread, extremely symptomatic lesions of tinea and suffer from frequent relapses after weeks of stopping treatment leading to significant impairment in quality of life. ${ }^{3}$ Terbinafine is considered drug of choice for dermatophytosis with favourable pharmacokinetic and mycological profile. Recently, there is an increase in the incidence of terbinafine resistance with increasing numbers of clinical failures and relapses when given in standard dose. ${ }^{4}$

\section{Objective of the study}

Due to absence of proper treatment guidelines for the management of recurrent corporis and cruris, dermatologists are using various combinations of oral antifungals, higher doses, longer duration which is nothing but hit and trial method rather than evidence-based approach. Therefore, this study was conducted to compare different terbinafine regimens to establish which regimens reduces the treatment failure rates and is safe and efficacious. 


\section{METHODS}

This was a prospective, randomised, parallel group, open label comparative pilot study and was conducted from January 2019-January 2020 (for a period of one year) in department of pharmacology, Pt. B. D. Sharma PGIMS, Rohtak in collaboration with department of skin, VD and leprosy, Pt. B.D Sharma PGIMS, Rohtak. Ethical clearance was taken from institutional ethical committee before starting the study.

\section{Sample size}

Due to lack of previous research on recurrent tinea corporis and cruris sample size was taken to be 60 according to prevalence in department of skin, VD and leprosy and study was taken as pilot study.

Patients with age between 15-60 years with recurrence of tinea corporis and cruris within 6 weeks after completion of the antifungal treatment with drugs other than terbinafine were included in the study. Patients were excluded from the study if they were suffering from infection other than tinea corporis and cruris or any other skin disease, or were intolerant to terbinafine, or had any serious concomitant illness, or if they had hepatic and renal diseases. Patients who met above mentioned inclusion criteria and were willing to sign informed consent were further examined mycologically for fungal elements in skin scrapings. Patients who were found to be positive for fungus on $\mathrm{KOH}$ wet mount were then enrolled in the study and were randomised into three groups (Group A: Double dose of terbinafine, group B: Double duration, group C: Control group). Total 90 patients were screened, 23 patients did not fit the inclusion criteria and 67 patients were randomised into three groups among which 5 patients were lost to follow up and 2 patients left study due to adverse effects. 60 patients completed the study. Patients were assessed for physician assessment 4-point scale (PA4PS). ${ }^{5}$ This scale was assessed in terms of sign and symptoms namely erythema, scaling and pruritis at 0,2, 4and 6-weeks sign and symptoms were scored from 0 to 3 where 0 being absent and 3 being severe. Skin scrapings were collected at 0, 2 and 4 weeks for wet $\mathrm{KOH}$ mount. Patients were monitored for any adverse drug reaction throughout the study and blood samples were collected for biochemical parameters testing like liver function test and renal function test at baseline and end of treatment period.

\section{Statistical analysis}

The data was coded and entered into Microsoft excel spreadsheet. Analysis was done using SPSS version 20 (IBM SPSS Statistics Inc., Chicago, Illinois, USA) Windows software program. Descriptive statistics included computation of percentages, means and standard deviations. The analysis of variance (ANOVA) [for quantitative data within three groups] with post hoc Bonferroni test (intra-group comparison) were used for quantitative data comparison of all clinical indicators. Chi- square test and fisher exact test were used for qualitative data whenever 2 or more than 2 groups were used to compare. Level of significance was set at the $p$ value of 0.05 .

\section{RESULTS}

In this study 90 patients were screened among which 67 patients were recruited into three groups. Sixty patients completed the study. Participants were between 18-60 years of age among which 32 were males and 28 were females. In PA4P scale maximum reduction in erythema was seen in group with longer duration of terbinafine i.e., from $93.4 \%$ at the end of 6 weeks from baseline and was statistically significant whereas in group with double dose erythema reduced by $76.9 \%$ from baseline and in standard treatment group erythema reduced by $46.3 \%$ from baseline at the end of 6 weeks. Pruritis was maximally reduced in group with longer duration $93.18 \%$ from baseline at the end of week 6 and was statistically significant $(\mathrm{p}<0.05)$. However, group with double dose showed $65.78 \%$ reduction in pruritis at the end of 6 weeks and group with standard treatment showed reduction of $15 \%$ in pruritis at the end of 6 weeks as compared to baseline. Group with longer duration showed statistically significant $(p<0.05)$ reduction in scaling at the end of 4 weeks and 6 weeks i.e., $80 \%$ and $91.4 \%$ respectively. Group with double dose showed $59.2 \%$ reduction in scaling at the end of 6 weeks and group with standard treatment showed minimum reduction in scaling with $29.4 \%$ reduction. While assessing composite physician assessment four-point scale maximum reduction was seen in group with longer duration of treatment at the end of week 4 and week 6 which was statistically significant $(\mathrm{p}<0.05)$. While in group with double dose there was $58.1 \%$ and $46.5 \%$ reduction at week 4 and week 6 . And group with standard treatment had minimum reduction with $25 \%$ and $20.4 \%$ reduction at the end of week 4 and week 6 respectively as shown in Table 1.

As shown in Table 2, mycologically all patients were negative at baseline while at two weeks there were $90 \%$, $80 \%$, and $95 \%$ patients with negative smears at the end of two weeks in double dose group, longer duration group and group with standard therapy respectively. While at the end of 4 weeks group with longer duration showed $100 \%$ patients had negative smears, group with double dose showed $80 \%$ patients exhibiting negative smears and group with standard therapy showed $50 \%$ patients having negative smears.

Safety was assessed throughout the period of 6 weeks by observing patients for side effects like skin irritation, burning sensation, dryness of skin, pustules, folliculitis etc. Two patients in double dose group developed mild to moderate hot flushes and were termed as probable on causality assessment while reporting on adverse drug reaction. The laboratory parameters were all within normal limits in all treatment groups and no statistical significance seen as shown in Table 3. 
Table 1: Assessment of composite physician assessment 4-point scale (pa4ps) in different treatment groups.

\begin{tabular}{|c|c|c|c|c|c|c|c|}
\hline \multirow{2}{*}{ PA4PS } & \multicolumn{2}{|c|}{$\begin{array}{l}\text { Terbinafine ( } 500 \mathrm{mg} \text { OD* } 2 \\
\text { weeks), Group } \mathrm{A},(\mathrm{n}=20)\end{array}$} & \multicolumn{2}{|c|}{$\begin{array}{l}\text { Terbinafine ( } 250 \text { mg OD* } 4 \\
\text { weeks), Group B, (n=20) }\end{array}$} & \multicolumn{2}{|c|}{$\begin{array}{l}\text { Terbinafine ( } 250 \mathrm{mg} \text { OD* } 2 \\
\text { weeks), Group } \mathrm{C},(\mathrm{n}=20)\end{array}$} & \multirow{2}{*}{$P$ value } \\
\hline & Mean \pm SD & $\begin{array}{l}\% \text { Change } \\
\text { from baseline }\end{array}$ & Mean \pm SD & $\begin{array}{l}\% \text { Change } \\
\text { from baseline }\end{array}$ & Mean \pm SD & $\begin{array}{l}\% \text { Change } \\
\text { from baseline }\end{array}$ & \\
\hline $\begin{array}{l}\text { At } \\
\text { Baseline }\end{array}$ & $2.15 \pm 0.489$ & - & $2.40 \pm 0.503$ & - & $2.20 \pm 0.616$ & - & 0.3 \\
\hline At week 2 & $1.25 \pm 0.550$ & $0.9-(41.8)$ & $1.25 \pm 0.550$ & $1.15-(47.9)$ & $1.10 \pm 0.553$ & $1.1-(50)$ & 0.61 \\
\hline At week 4 & $0.90 \pm 0.968$ & $1.25-(58.1)$ & $0.50 \pm 0.513$ & $1.9-(79.1)$ & $1.65 \pm 0.745$ & $0.55-(25)$ & $<0.001$ \\
\hline At week 6 & $1.15 \pm 0.933$ & $1-(46.5)$ & $0.10 \pm 0.308$ & $2.3-(95.8)$ & $1.75 \pm 0.786$ & $0.45-(20.4)$ & $<0.001$ \\
\hline
\end{tabular}

Table 2: Number of patients with negative microscopic examination (mycological cure) in different treatment groups.

\begin{tabular}{|c|c|c|c|c|}
\hline Variables & $\begin{array}{l}\text { Terbinafine (500 mg OD* } 2 \\
\text { weeks), Group } \mathrm{A},(\mathrm{n}=20)\end{array}$ & $\begin{array}{l}\text { Terbinafine ( } 250 \mathrm{mg} \text { OD* } 4 \\
\text { weeks), Group B, }(\mathrm{n}=20)\end{array}$ & $\begin{array}{l}\text { Terbinafine ( } 250 \mathrm{mg} \mathrm{OD}^{*} 2 \\
\text { weeks), Group } \mathrm{C},(\mathrm{n}=20)\end{array}$ & $\begin{array}{l}\mathbf{P} \\
\text { value* }\end{array}$ \\
\hline Baseline & 0 & 0 & 0 & NA \\
\hline 2 weeks & $18(90)$ & $16(80)$ & $19(95)$ & 0.074 \\
\hline 4 weeks & $16(80)$ & $20(100)$ & $10(50)$ & 0.030 \\
\hline
\end{tabular}

Table 3: Assessment of laboratory parameters in different treatment groups.

\begin{tabular}{|llllllllll|}
\hline Variables & \multicolumn{2}{l}{ Group A (weeks) } & \multicolumn{3}{l}{ Group B (weeks) } & \multicolumn{2}{ll}{ Group C (weeks) } \\
& $\mathbf{0}$ & $\mathbf{2}$ & $\mathbf{P}$ & $\mathbf{0}$ & $\mathbf{4}$ & $\mathbf{P}$ & $\mathbf{0}$ & $\mathbf{2}$ & P \\
\hline AST & $82.30 \pm 12.28$ & $82.90 \pm 11.62$ & 0.15 & $38.55 \pm 8.91$ & $40 \pm 8.97$ & 0.35 & $41.90 \pm 9.85$ & $43.00 \pm 9.36$ & 0.47 \\
\hline ALT & $30.95 \pm 7.70$ & $31.85 \pm 6.42$ & 0.22 & $34.70 \pm 6.48$ & $35.90 \pm 6.57$ & 0.24 & $34.25 \pm 5.12$ & $36.40 \pm 5.49$ & 0.45 \\
\hline ALP & $28.55 \pm 5.99$ & $29.30 \pm 5.62$ & 0.21 & $33.60 \pm 7.65$ & $34.30 \pm 6.83$ & 0.11 & $32.70 \pm 5.86$ & $33.25 \pm 5.07$ & 0.43 \\
\hline Urea & $24.00 \pm 4.43$ & $24.60 \pm 4.68$ & 0.05 & $32.35 \pm 8.36$ & $32.60 \pm 7.74$ & 0.42 & $33.15 \pm 9.06$ & $32.95 \pm 8.42$ & 0.64 \\
\hline Creatinine & $0.83 \pm 0.08$ & $0.82 \pm 0.07$ & 0.64 & $0.77 \pm 0.12$ & $0.77 \pm 0.12$ & 1 & $0.72 \pm 0.13$ & $0.73 \pm 0.14$ & 0.78 \\
\hline Protein & $7.93 \pm 0.94$ & $7.98 \pm 0.93$ & 0.28 & $7.18 \pm 1.12$ & $7.29 \pm 0.97$ & 0.13 & $7.56 \pm 1.25$ & $7.56 \pm 1.27$ & 1 \\
\hline
\end{tabular}

\section{DISCUSSION}

In this study efficacy was assessed by PA4PS which assesses the affectivity of the treatment based on the evaluation of signs and symptoms like erythema, pruritis and scaling and it was seen that treatment group with double duration gave maximum relief in erythema, pruritis, and scaling at the end of week 4 and week 6 which is in accordance with below mentioned studies.

In a study by Kumar et al there was significant decrease in the clinical score beginning from baseline to $4^{\text {th }}$ week in both, terbinafine in longer duration and fluconazole groups $(p<0.05) .{ }^{6}$ If we compare the clinical score of both the groups after 4 week there was slightly more reduction of clinical score in group with longer duration of terbinafine than of group with fluconazole. Results of this study was found to be similar to our study as terbinafine in longer duration i.e., 4 week was found to be more efficacious.

Results obtained from our study could be due to the fact that the therapeutic effect of terbinafine in the stratum corneum remained for 2-3 weeks after stopping therapy. This is based on the skin pharmacokinetics of the major drugs used in dermatophytosis. One of the principal reasons of decreased efficacy is decreased effective drug concentration, which might be in case of terbinafine following standard dosing regimen of $250 \mathrm{mg}$ daily due to extensive accumulation in skin and adipose tissue. Although when terbinafine is given for longer durations, it remains in stratum corneum for longer period of time after stopping the drug which explains better efficacy.

On assessing mycological efficacy in this study through $\mathrm{KOH}$ wet mount it was seen that at the end of 4 weeks, terbinafine when given for double duration group had better results in terms of acquiring mycological cure as compared to double dose group and control group. This result is in accordance with a survey done by Babu et al where he observed efficacy and safety of terbinafine 500 $\mathrm{mg}$ in 2 weeks, 4 weeks and 6 weeks in patients of dermatophytosis. ${ }^{4}$ Among all recruited patients, $\mathrm{KOH}$ and culture positivity was recorded in $80 \%$ and $65.5 \%$ of cases respectively and mycological cure rates were better when terbinafine was given for 4 weeks. Also, in a study done by Kumar et al, where he compared efficacy of terbinafine and fluconazole in patients of tinea corporis, clinical response rate was calculated as healed (negative $\mathrm{KOH}$ and no signs and symptoms) or markedly improved (negative $\mathrm{KOH}$ with signs and symptoms). ${ }^{6}$ The clinical response 
rate was more in group with terbinafine when given for 4 weeks as compared to fluconazole.

In a study by Bhatia et al where she compared efficacy of oral terbinafine (500 $\mathrm{mg}$ OD for 4 weeks) versus itraconazole (200 $\mathrm{mg}$ OD for 4 weeks) in treatment of dermatophyte infection of skin, the absolute score of erythema, scaling, and pruritus was significantly different at baseline when both the groups were compared. ${ }^{7}$ Hence, they calculated the percentage change in the scores of all three parameters. The percentage change was significantly more in pruritus from 2 to 4 weeks only in group I. In group II, the percentage change in pruritus and scaling was significantly different from 0 to 2 weeks and 2-4 weeks. On comparing the groups, there was a significant improvement in scaling score from 0 to 2 weeks and from 2 to 4 weeks but not from 0 to 4 weeks. There was a significant improvement in pruritus from 2 to 4 weeks only. There was no statistically significant percentage change in erythema scores. Results of this study do not match with our study. Reasons might be shorter duration of follow-up and patients included in the study were not suffering from recurrent tinea. In this study it was also established that terbinafine in higher dose is more efficacious with lesser relapses.

Also, at baseline and at the end of treatment, patients were assessed for biochemical parameters like liver function test and renal function tests. All the lab parameters were within range and showed insignificant difference in all the groups. Hence, it can be said that terbinafine is safe when given for higher doses or longer duration. In a study by Sirohi et al done to compare safety, efficacy and quality of life in patients in oral terbinafine and amphotericin B with topical and sertaconazole cream for the treatment of tinea corporis there was no serious adverse event reported in both groups. ${ }^{8}$ None of the patients required reduction in dose or any therapy for treatment of adverse events. No patient was lost to the follow up. Results of our study are in accordance with other studies mentioned above as terbinafine was found to be safe.

\section{Limitations}

Cultures could not be done due to reluctance of patients for getting cultures done keeping in view the longer duration of time required for the culture results.

\section{CONCLUSION}

Terbinafine when given in longer duration is safe, more efficacious as compared to increased dose or standard therapy in patients of recurrent tinea corporis and cruris. In conclusion, in absence of any consensus on treatment of recurrent tinea corporis and cruris, terbinafine when given in longer duration provides more efficacious and safe treatment option according to our study.

Funding: No funding sources

Conflict of interest: None declared

Ethical approval: The study was approved by the Institutional Ethics Committee

\section{REFERENCES}

1. Rajagopalan M, Inamdar A, Mittal A, Miskeen AK, Srinivas CR, Sardana K et al. Expert consensus on the management of dermatophytosis in India (Ectoderm India). BMC Dermatol. 2008;18:6.

2. Sharma Y, Jain S, Chandra K, Khurana VK, Kudesia M. Clinico-mycological evaluation of dermatophytes and non-dermatophytes isolated from various clinical samples: A study from north India. J Res Med Sci. 2012;17:817-8.

3. Dogra S, Uprety S. The menace of chronic and recurrent dermatophytosis in India: Is the problem deeper than we perceive? Indian Dermatol Online J. 2016;7(2):73-6.

4. Babu PR, Pravin AJS, Deshmukh G, Dhoot D, Samant A, Kotak B. Efficacy and Safety of Terbinafine 500 mg once daily in patients with dermatophytosis. Indian J Dermatol. 2017;62:395-9.

5. Banerjee M, Ghosh AK, Basak S, Das KD, Gangopadhyay DN. Comparative evaluation of effectivity and safety of topical amorlfine and clotrimazole in the treatment of tinea corporis. Indian J Dermatol. 2011;56(6):657-62.

6. Kumar A, Budania N, Sharma P, Singh M. A comparative study of efficacy of terbinafine and fluconazole in patients of tinea corporis. Int J Biomed Res. 2013;04:11.

7. Bhatia A, Kanish B, Badyal DK, Kate P, Choudhary S. Efficacy of oral terbinafine versus itraconazole in treatment of dermatophytic infection of skin-A prospective, randomized comparative study. Indian J Pharmacol. 2019;51:116-9.

8. Sirohi S, Bhutani S, Cheema C, Kaur G, Singh SP. Comparison of safety and efficacy of oral terbinafine with amphotericin B gel and sertaconazole cream for the treatment of tinea corporis and its effect on quality of life of patients. Int $\mathrm{J}$ Dermatol Clin Res. 2017;3(1):18-21.

Cite this article as: Verma N, Verma S, Dayal S, Gupta MC. Efficacy and safety of different terbinafine regimens in patients of recurrent tinea corporis and cruris. Int J Basic Clin Pharmacol 2021;10:911-4. 